\title{
Effect of P lumbago zeylanica administration on brain neurotransmitter leve in Wistar albino rats
}

\author{
Muthukumarasamy Baskaran ${ }^{1}$, Latha Shanmugam², Vijayakumar Raman ${ }^{2} *$ \\ ${ }^{1}$ Department of General Medicine, Sri Lakshmi Narayana Institute of Medical Sciences, Puducherry-605 502, India. \\ ${ }^{2}$ Department of Physiology, Sri Lakshmi Narayana Institute of Medical Sciences, Puducherry-605 502, India.
}

\begin{tabular}{l} 
ARTICLE INFO \\
\hline Article history: \\
Received on: $27 / 10 / 2014$ \\
Revised on: $18 / 12 / 2014$ \\
Accepted on: 16/02/2015 \\
Available online: $27 / 04 / 2015$ \\
\hline Key words: \\
Plumbago zeylanica, \\
Naphthoquinone, Plumbagin, \\
Brain neurotransmitter, \\
HPLC.
\end{tabular}

\begin{abstract}
According to WHO about $80 \%$ of the world's population relies on traditional medicine for their primary health care. Plumbago zeylanica L. is a medicinal plant, belong to the family of Plumbaginaceae and the root of $P$. zeylanica contains several bioactive constituent like L-dopa, Plumbagin (naphthoquinone), droseron, chitranone, triterpenoid, anthraquinone. This study is designed to evaluate the effect of $P$. zeylanica and naphthoquinone on the level of various amine neurotransmitters namely epinephrine, norepinephrine, serotonin, 5-hydroxyindole 3acetic acid (5-HIAA), and dopamine on the discrete regions of the brain tissues. Wistar male albino rats were treated separately with ethanoloic extract of $P$. zeylannica (root) and the commercially purchased phytochemical naphthoquinone at the dose of $2 \mathrm{mg} / \mathrm{kg}$ b. wt with different experimental groups. The brain tissue homogenates (cerebral cortex, cerebellum, hypothalamus, pons-medulla, midbrain, and corpus striatum) were analyzed to quantify the aforementioned neurotransmitters by high performance liquid chromatography (HPLC). The results showed that, administration of P. zeylanica and NQ does not alter the many of the studied neurotransmitter at significant level; however, there is a change in the neurotransmitter profile in few specific regions of Wistar rat brain and striatum was found to be affected more.
\end{abstract}

\section{INTRODUCTION}

Today the scientists of the world are visualizing a great future of plant based drugs for treatment of cancer, AIDS, chronic diseases and viral infections, since plants appear to be an excellent source of new bioactive compounds. Therefore, a worldwide interest is developing rapidly in phytomedicinal sector. Plumbago zeylanica L. is a medicinal plant, belong to the family of Plumbaginaceae. Plumbago zeylanica (PZ) is an attractive erect rambling shrub with long tuberous root and often occurring as a roadside weed, sometimes cultivated in gardens. PZ is being used for the cure of skin diseases, infections and intestinal worms such as scabies, hookworm dermatitis, acne, ulcers, sores, ringworm and hookworm (Manu pant, 2012). The main constituent in $\mathrm{PZ}$ is plumbagin, which forms 50. 27 parts in one gram (De Paiva et al., 2003). Siva Kumar et al., (2005)

* Corresponding Author

Email:slimspub@yahoo.co.in reported that the plumbagin $\mathrm{LD}_{50}$ dose was found to be $16 \mathrm{mg} / \mathrm{kg}$ body weight in mice. However, according to Santhakumari and Rathinam (1978), it is $6.5 \mathrm{mg} / \mathrm{kg}$ body weight in albino rats. The Plumbagin, a quinonoid constituent isolated from the root of PZ has been shown to exert anti carcinogenic effect (Srinivas et al., 2004). PZ exhibits an inhibitory effect on carcinogenesis in the intestines, causes cytogenetic and cell cycle changes in mouse and it is also effective in Ehrlich ascites carcinoma, and possesses proliferation activity in human cervical cancer cells (Srinivas et al., 2004). PZisalso reported to have antitumour activity on rat fibrosarcoma (Krishnaswamy and Purushothaman, 1980). Plumbagin, however, showed exceptional antimutagenicity (Edenharder and Tang, 1997). Plumbagin has demonstrated reproductive toxicity in male and female animals. Extracts of the root have been reported to be a powerful poison which, when given internally or applied to the ostium uteri, causes abortion (Premakumari et al., 1977). Krishnaswamy and Purushothaman, (1980) reported that Plumbagin has antimicrobial and antifungal activities. 
Abdul and Ramchender, (1995) also reported that in low doses plumbagin exerted a constant increase in bactericidal activity throughout the study period whereas with a high dose a higher response was observed up to six weeks.

\section{MATERIALS AND METHODS}

The chemicals norepinephrine, Epinephrine, 5hydroxytryptamine (5-HT), 5-hydroxyindole 3-acetic acid (5HIAA), 3,4, dihydroxy benzyl amine hydrobromide (DHBA) and naphthoquinone were purchased from Sigma. Other chemicals were of analytical grade from Merk (Germany) and SISCO (India).

\section{Animals}

Wistar strain male albino rats weighing 180 to $220 \mathrm{~g}$ were used for all the animal experiments. Animals were housed in groups of three (rats) per cage and maintained in the animal house. The animals were kept under closely controlled environmental conditions (12-hr light/dark cycle, lights on between 07.00 and 19. $00 \mathrm{hr}$, room temperature, $24^{\circ} \mathrm{C}$ ) and allowed free access to food (M/S Hindustan Lever Limited, Bombay, India) and water. The study commenced after obtaining the approval from the Institutional Animal Ethical Committee and Committee for Purpose of Control and Supervision of Experiments on Animals (CPCSEA), Government of India. All efforts were made to minimize both the number of animals used and unwanted stress or discomfort to the animals during experimental procedures. All the sample collections were done between 07. 00 and 09. 00AM in order to avoid circadian rhythm induced variation.

\section{Plant and Extraction}

The root of the Plumbago zeylanica (PZ) was collected locally, identified and authenticated by the Tamil Nadu Medicinal Plant Farms \& Herbal Medicine Corporation Ltd. , Chennai, Tamil Nadu - 600 106. The extraction procedure was done according to Bopaiah and Pradhan, (2001). The dried coarsely powdered root of PZ (50g) was extracted with $50 \%$ ethanol using a Soxhlet extractor for $24 \mathrm{~h}$ at constant temperature $\left(50-55^{\circ} \mathrm{C}\right)$. Then the extract was concentrated in a water bath under reduced pressure. The semisolid form of the extract was dried in hot air oven. The suspension of the extract was prepared in Olive oil (1:9). Naphthoquinone (NQ) was purchased from Sigma. The ethanolic extract of PZ root as well as the NQ was administered orally by dissolving it in olive oil (1:9) at the dosage of $2 \mathrm{mg} / \mathrm{kg}$ body weight (Santhakumari and Rathinam, 1978).

\section{Experimental Groups}

Experimental animals were divided into 8 groups, each consists 6 animals. Group I animals were control animals (untreated), Group II animals were treated with Olive oil orally. Group III, IV and V animals were treated orally $(2 \mathrm{mg} / \mathrm{kg} \mathrm{b}$. wt) with ethanolic extract of PZ root for 1day, 15days, and 30 days respectively. Group VI, VII and VIII animals were treated NQ ( $2 \mathrm{mg} / \mathrm{kg} \mathrm{b}$. wt)orally for 1 day, 15 days, and 30 days respectively.

\section{Sample preparation}

At the end of the experiment, animals were killed by cervical dislocation and the brain was dissected, discrete regions namely cerebral cortex (CC), cerebellum (CB), midbrain (MB), pons-medulla (PM), hippocampus (HP) and hypothalamus (HT) were micro dissected and individual brain regions were homogenized separately using motor driven Teflon-glass tissue homogenizer with $60 \mathrm{mg} / \mathrm{ml}$ of ice-cold $0.1 \mathrm{M}$ perchloric acid. Homogenates were centrifuged at $6,000 \mathrm{~g}$ in a refrigerated centrifuge for $2 \mathrm{~min}$. The supernatant $600 \mu \mathrm{l}$ was taken in an eppendorf to which dihydroxy benzyl hydrazine (DHBA) $20 \mu \mathrm{l}$ (200pg) was added as an internal standard. Again, it was centrifuged at $35,000 \mathrm{~g}$ for 20 minutes. Supernatant was filtered with $0.22 \mu$ membrane filter.

\section{Estimation of brain biogenic amines}

The various brain biogenic amines in the discrete regions of the rat brain were estimated by the method of Wagner et al., (1982). Concentrations of Norepinephrine (NE), Epinephrine (E), 5-hydroxytryptamine (5-HT) and 5-hydroxyindole 3-acetic acid (5-HIAA) were measured by high performance liquid chromatography (HPLC) coupled with electrochemical detection as per the method of Kim et al., (1983). Separation and quantization were achieved by reverse phase HPLC column with electrochemical detection.

One $\mathrm{mg}$ of NE, E, 5HT and 5-HIAA, were dissolved separately in $10 \mathrm{ml}$ of $0.1 \mathrm{M}$ perchloric acid containing $0.05 \mathrm{mM}$ ascorbic acid initially and ran in the HPLC system to determine the retention time of the individual sample. Then all were mixed to make a final concentration to run. No difference in the retention time was observed for the individual neurotransmitter. The mixed solution was stored as stock solution at $-70^{\circ} \mathrm{C}$ and was freshly prepared once in every two weeks. Just before use, $10 \mu \mathrm{l}$ of each stock solution (containing all the above) was taken and made up to $100 \mathrm{ml}$ with distilled water. $20 \mu \mathrm{l}$ from this was injected into HPLC, which contains 200pg of all standards. DHBA was used as internal standards.

Twenty micro liter of sample was injected into a Rheodyne injector (USA) of HPLC system, which was connected to an isocratic pump and reverse phase column for separation of indole amines and catecholamines (Figure 9). The reaction products were detected with electrochemical detector (ECD). The electrochemical detector (ECD) with glassy carbon working electrode was used at a voltage setting of +0 . $60 \mathrm{~V}$ for monoamines and their metabolites vs. an $\mathrm{Ag} / \mathrm{AgCl}$ reference electrode. The flow rate was maintained at $0.8 \mathrm{ml} / \mathrm{min}$. Neurotransmitters were quantified using Shimadzu C-R8A data processor and expressed as nano grams of neurotransmitter per gram of wet weight of brain tissue.

Concentrations of Homovalinic acid (HVA), Dopamine (DA), 3,4, dihydroxy benzyl acetic acid (DOPAC) were measured by HPLC coupled with electrochemical detection as per the system specification above. 


\section{RESULTS}

\section{Epinephrine (E)}

The epinephrine from various groups is summarized in table no-1as mean \pm SEM. One-day treatment with PZ or NQ didn't alter the epinephrine level all in the brain regions studied. After 15 days treatment PZ did not alter the epinephrine level in any of the regions whereas NQ treatment increased the epinephrine level only in striatum $(\mathrm{F}=5$, df 7, 40). However, the rest of the regions remain unaffected. The 30 days of PZ and NQ treatment none of the region showed variation. At this dosage, PZ even after repeated exposure did not interfere with the epinephrine.

\section{Norepinephrine (NE)}

The norepinephrine from various groups is summarized in table no. 2 as mean \pm SEM. One-day treatment with PZ or NQ didn't alter the norepinephrine level in the brain. After 15 days treatment with both PZ and NQ treatment increased the norepinephrine level in striatum $(F=9.2$, df 7, 40). However, the rest of the regions remain unaffected. The 30 days of PZ and NQ treatment none of the region showed variation.
At this dosage, PZ even after repeated exposure did not interfere with the norepinephrine.

\section{Serotonin (5 HT)}

The Serotonin from various groups is summarized in table no - 3as mean \pm SEM. One-day treatment with PZ or NQ didn't alter the serotonin level all in the brain regions studied. After 15 days treatment with PZ didn't alter the serotonin level in all the regions studied. , whereas with NQ treatment showed increased serotonin level in cerebellum $(F=5.6$, df 7, 40). However, the rest of the regions remain unaffected. The 30 days of $\mathrm{PZ}$ and NQ treatment increased the serotonin level in striatum $(\mathrm{F}=$ 7.7, df 7, 40). However, the rest of the regions remain unaffected. At this dosage, $\mathrm{PZ}$ even after repeated exposure did not interfere with the serotonin.

\section{5-Hydroxy Indole Acetic acid}

The 5 HIAA from various groups is summarized in table no-4as mean \pm SEM. Irrespective of the duration PZ or NQ administration, the levels of the 5 HIAA did not show any variation from controls indicating (one day, 15 days and 30 days) both of them are not interfering with the normal functions of brain.

Table 1: Effect of PZ and NQ treatment on epinephrine level in brain tissue.

\begin{tabular}{|c|c|c|c|c|c|c|c|c|}
\hline & \multicolumn{8}{|c|}{ EPINEPHRINE (ng/g of wet tissue) } \\
\hline & CONTROL & VEHICLE & PZ 1 & PZ 15 & PZ 30 & NQ 1 & NQ 15 & NQ 30 \\
\hline$\overline{\mathrm{CC}}$ & $125 \pm 3$ & $121 \pm 5$ & $108 \pm 5$ & $117 \pm 5$ & $125 \pm 5$ & $109 \pm 8$ & $114 \pm 7$ & $118 \pm 3$ \\
\hline CB & $1460 \pm 77$ & $1397 \pm 53$ & $1307 \pm 63$ & $1305 \pm 33$ & $1394 \pm 97$ & $1415 \pm 68$ & $1433 \pm 76$ & $1367 \pm 92$ \\
\hline HY & $1864 \pm 95$ & $1486 \pm 51$ & $1421 \pm 116$ & $1692 \pm 106$ & $1663 \pm 104$ & $1596 \pm 93$ & $1581 \pm 77$ & $1775 \pm 133$ \\
\hline HP & $5774 \pm 520$ & $5266 \pm 497$ & $5592 \pm 323$ & $5658 \pm 580$ & $6049 \pm 369$ & $5399 \pm 432$ & $5424 \pm 307$ & $5576 \pm 345$ \\
\hline PM & $5824 \pm 318$ & $5437 \pm 159$ & $6096 \pm 346$ & $5398 \pm 349$ & $4920 \pm 348$ & $5238 \pm 256$ & $5613 \pm 490$ & $5487 \pm 359$ \\
\hline ST & $2056 \pm 92$ & $1889 \pm 110$ & $1982 \pm 98$ & $2274 \pm 33$ & $2019 \pm 36$ & $1934 \pm 137$ & $2522 \pm 60 *$ & $2094 \pm 107$ \\
\hline
\end{tabular}

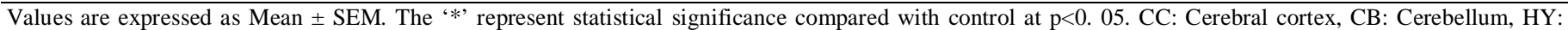

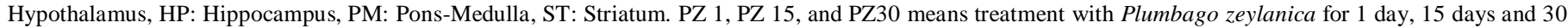
days respectively. NQ 1, NQ 15 and NQ 30 means treatment with naphthoquinone respectively for 1 day, 15 days and 30 days.

Table 2: Effect of PZ and NQ treatment on nor-epinephrine level in brain tissue.

\begin{tabular}{|c|c|c|c|c|c|c|c|c|}
\hline & \multicolumn{8}{|c|}{ NOREPINEPHRINE (ng/g of wet tissue) } \\
\hline & CONTROL & VEHICLE & PZ 1 & PZ 15 & PZ 30 & NQ 1 & NQ 15 & NQ 30 \\
\hline$\overline{\mathrm{CC}}$ & $777 \pm 39$ & $654 \pm 36$ & $711 \pm 36$ & $668 \pm 47$ & $676 \pm 25$ & $759 \pm 50$ & $732 \pm 37$ & $796 \pm 44$ \\
\hline $\mathrm{CB}$ & $1862 \pm 125$ & $157 \pm 106$ & $1836 \pm 148$ & $1790 \pm 91$ & $1655 \pm 117$ & $1679 \pm 76$ & $1761 \pm 102$ & $1854 \pm 100$ \\
\hline HY & $25165 \pm 1206$ & $23016 \pm 1280$ & $26078 \pm 1049$ & $240613 \pm 980$ & $22665 \pm 823$ & $24801 \pm 1655$ & $24996 \pm 1601$ & $24265 \pm 1140$ \\
\hline HP & $7817 \pm 455$ & $6529 \pm 520$ & $7778 \pm 312$ & $7757 \pm 468$ & $7817 \pm 455$ & $7554 \pm 422$ & $6301 \pm 2726$ & $6671 \pm 602$ \\
\hline PM & $4200 \pm 339$ & $4390 \pm 344$ & $4123 \pm 263$ & $4687 \pm 388$ & $3919 \pm 223$ & $4495 \pm 322$ & $4707 \pm 305$ & $4509 \pm 242$ \\
\hline $\mathrm{ST}$ & $9482 \pm 468$ & $9376 \pm 569$ & $9817 \pm 278$ & $13872 \pm 781 *$ & $10200 \pm 592$ & $10207 \pm 580$ & $12550 \pm 546 *$ & $9577 \pm 407$ \\
\hline
\end{tabular}

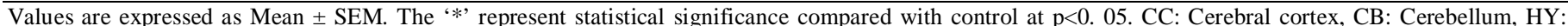

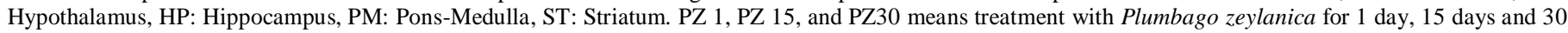
days respectively. NQ 1, NQ 15 and NQ 30 means treatment with naphthoquinone respectively for 1 day, 15 days and 30 days.

Table 3: Effect of PZ and NQ treatment on serotonin level in brain tissue.

\begin{tabular}{|c|c|c|c|c|c|c|c|c|}
\hline & \multicolumn{8}{|c|}{ SEROTONIN (ng/g of wet tissue) } \\
\hline & CONTROL & VEHICLE & PZ 1 & PZ 15 & PZ 3 & NQ 1 & NQ 15 & NQ 30 \\
\hline$\overline{\mathbf{C C}}$ & $783 \pm 30$ & $793 \pm 44$ & $683 \pm 31$ & $822 \pm 37$ & $735 \pm 47$ & $805 \pm 36$ & $825 \pm 38$ & $758 \pm 42$ \\
\hline CB & $893 \pm 39$ & $929 \pm 34$ & $794 \pm 35$ & $822 \pm 33$ & $762 \pm 34$ & $730 \pm 33$ & $1022 \pm 42 *$ & $791 \pm 39$ \\
\hline HY & $8954 \pm 440$ & $9228 \pm 427$ & $9362 \pm 445$ & $9284 \pm 415$ & $9876 \pm 489$ & $9848 \pm 477$ & $9340 \pm 469$ & $9225 \pm 421$ \\
\hline HP & $4045 \pm 196$ & $4057 \pm 207$ & $4212 \pm 199$ & $3977 \pm 200$ & $3974 \pm 157$ & $3930 \pm 203$ & $3756 \pm 202$ & $3941 \pm 146$ \\
\hline PM & $2646 \pm 212$ & $2235 \pm 186$ & $2944 \pm 207$ & $2901 \pm 151$ & $2617 \pm 190$ & $3356 \pm 174$ & $2948 \pm 231$ & $3049 \pm 229$ \\
\hline ST & $5550 \pm 140$ & $5078 \pm 409$ & $5597 \pm 254$ & $5797 \pm 200$ & $7400 \pm 299 *$ & $5112 \pm 382$ & $5192 \pm 294$ & $7297 \pm 289 *$ \\
\hline
\end{tabular}

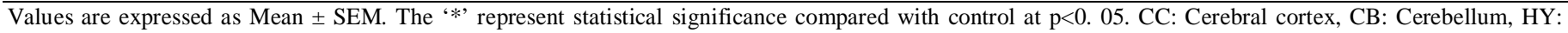

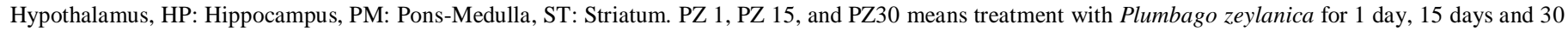
days respectively. NQ 1, NQ 15 and NQ 30 means treatment with naphthoquinone respectively for 1 day, 15 days and 30 days. 
Table 4: Effect of PZ and NQ treatment on serotonin level in brain tissue.

\begin{tabular}{llllllll}
\hline 5-HIAA & & \multicolumn{7}{c}{ 5-HYDROXY INDOLE ACETIC ACID (ng/g of wet tissue) } \\
\hline CC & CONTROL & VEHICLE & PZ 1 & PZ 15 & PZ 30 & NQ 1 & NQ 15 \\
CB & $63 \pm 4$ & $55 \pm 2$ & $65 \pm 3$ & $55 \pm 2$ & $60 \pm 3$ & $65 \pm 2$ & $64 \pm 3$ \\
HY & $141 \pm 12$ & $156 \pm 11$ & $158 \pm 11$ & $148 \pm 10$ & $125 \pm 6$ & $131 \pm 12$ & $136 \pm 9$ \\
HP & $986 \pm 84$ & $903 \pm 68$ & $1025 \pm 90$ & $1048 \pm 95$ & $987 \pm 55$ & $1021 \pm 51$ & $1039 \pm 73$ \\
PM & $345 \pm 27$ & $321 \pm 22$ & $306 \pm 17$ & $369 \pm 21$ & $429 \pm 21$ & $338 \pm 25$ & $351 \pm 25$ \\
ST & $389 \pm 30$ & $347 \pm 28$ & $353 \pm 20$ & $398 \pm 33$ & $418 \pm 27$ & $372 \pm 22$ & $439 \pm 30$ \\
\hline
\end{tabular}

Values are expressed as Mean \pm SEM. The '*' represent statistical significance compared with control at p<0. 05. CC: Cerebral cortex, CB: Cerebellum, HY: Hypothalamus, HP: Hippocampus, PM: Pons-Medulla, ST: Striatum. PZ 1, PZ 15, and PZ30 means treatment with Plumbago zeylanica for 1 day, 15 days and 30 days respectively. NQ 1, NQ 15 and NQ 30 means treatment with naphthoquinone respectively for 1 day, 15 days and 30 days.

Table 5: Effect of PZ and NQ treatment on the level of dopamine and its metabolites in brain tissue.

\begin{tabular}{|c|c|c|c|c|c|c|c|c|}
\hline & \multicolumn{8}{|c|}{ DOPAMINE (ng/g of wet tissue) } \\
\hline & $\mathrm{CON}$ & VEHICLE & PZ 1 & PZ 15 & PZ 30 & NQ 1 & NQ 15 & NQ 30 \\
\hline$\overline{\mathrm{CC}}$ & $689 \pm 60$ & $680 \pm 75$ & $694 \pm 67$ & $729 \pm 26$ & $715 \pm 140$ & $706 \pm 62$ & $920 \pm 33 *$ & $721 \pm 144$ \\
\hline $\mathbf{C B}$ & $1828 \pm 383$ & $1969 \pm 522$ & $2025 \pm 405$ & $2070 \pm 364$ & $2025 \pm 405$ & $2053 \pm 508$ & $2294 \pm 498$ & $1907 \pm 234$ \\
\hline HY & $4293 \pm 607$ & $4319 \pm 714$ & $4408 \pm 557$ & $5366 \pm 1021$ & $4608 \pm 595$ & $4733 \pm 1062$ & $5820 \pm 1328$ & $4833 \pm 1081$ \\
\hline HP & $1219 \pm 177$ & $1374 \pm 330$ & $1505 \pm 215$ & $1472 \pm 203$ & $1386 \pm 170$ & $1714 \pm 425$ & $1778 \pm 139 *$ & $1445 \pm 179$ \\
\hline PM & $676 \pm 75$ & $688 \pm 89$ & $674 \pm 79$ & $779 \pm 128$ & $675 \pm 78$ & $693 \pm 119$ & $935 \pm 328$ & $683 \pm 87$ \\
\hline ST & $6370 \pm 1065$ & $6318 \pm 832$ & $6184 \pm 1031$ & $7180 \pm 970$ & $6144 \pm 784$ & $6269 \pm 820$ & $8205 \pm 568 *$ & $6286 \pm 782$ \\
\hline
\end{tabular}

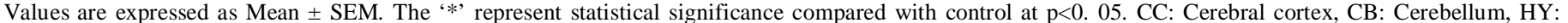

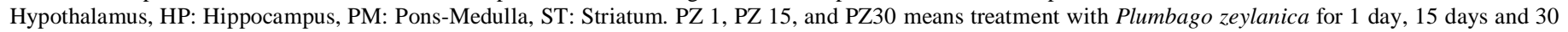
days respectively. NQ 1, NQ 15 and NQ 30 means treatment with naphthoquinone respectively for 1 day, 15 days and 30 days.

\section{Dopamine and its metabolites}

The dopamine, 3, 4 di-hydroxy phenyl acetic acid and homovanillic acid from various groups are summarized in figure 68-85 as well as in table 12,13 and 14 with mean \pm SEM. One-day treatment with PZ or NQ didn't alter the dopamine, DOPAC and HVA level in the brain. After 15 days treatment PZ did not alter the dopamine, DOPAC and HVA level in any of the regions whereas NQ treatment increased the dopamine level in cerebral cortex $(F=4$. 3, df 7, 40), hippocampus $(F=2$. 9, df 7,40) and striatum $(F=3.24$, df 7,40$)$. However, the rest of the regions remain unaffected. The 30 days of $\mathrm{PZ}$ and NQ treatment none of the region showed variation. At this dosage, $\mathrm{PZ}$ even after repeated exposure did not interfere with the dopamine, 3, 4 di-hydroxy phenyl acetic acid (DOPAC) and homovanillic acid.

\section{DISCUSSION}

There are reports available to support that the components in the herbs can cross blood brain barrier to affect the central nervous system. For instance, Ocimum sanctum (OS) can act as an anti-stressor. Further the anti-noise stressor activity of OS has already been reported for the central cholinergic system (Sembulingam et al., 2005). Kumar et al, (2002) reported that Indian Hypericum perforatum treatment (doses of 50 and 200 $\mathrm{mg} / \mathrm{kg}$ ) significantly decreased the levels of serotonin and its metabolite 5-hydroxy indole acetic acid and 5-HT turnover in all the brain regions (hypothalamus, hippocampus, striatum, ponsmedulla and frontal cortex). Moreover, they also reported that, it significantly augmented the levels of norepinephrine and its metabolite methylhydroxy phenyl glycol (MHPG) and norepinephrine turnover in all the brain regions studied. In this study, epinephrine (15 days with NQ) as well as norepinephrine (15 days with both NQ and PZ) increased in striatum whereas the dopamine increased in cerebral cortex, hippocampus and striatum (with NQ 15 days). Noteworthy that in spite of 30 days of repeated PZ and NQ administration most of the brain region showed no variation in most of the neurotransmitters studied. This may be due to habituation (a stimulus, when repeated over and over leads to the gradually disappearing response) which is associated with the decreased release of neurotransmitter from the pre synaptic terminal because of decreased intracellular calcium by inactivating calcium channels (Ganong, 2001).

The serotonin level in rats showed alteration in cerebellum (NQ fed 15 days) and in striatum (Both PZ and NQ fed) in this study. Though the level of serotonin increased no alteration in HPA activity was observed as indicated by the corticosteroid level probably these regions may not be involved in HPA axis regulation.

In the present study, the NQ as well as PZ during repeated exposure affected specifically the neurotransmitters in striatum. The effects of a $50 \%$ ethanol extract of the root of Plumbago zeylanica was investigated (Bopaiah and Pradham, 2001) on central nervous system in rats and showed an enhancement of the spontaneous ambulatory activity without inducing stereotypic behavior. In these animals the neuro chemical estimations by Bopaiah and Pradham, (2001) revealed an elevated level of dopamine and homovanillic acid in striatum compared to the control rats. From these results, they concluded the extract possess the stimulatory properties and this might be mediated by dopaminergic mechanisms in the rat brain. In this study, though PZ and NQ were used, the homovanillic acid level was not altered irrespective of duration and whether PZ or NQ. The possible reason behind this was they used a very high dose of PZ (100,200 and $300 \mathrm{mg} / \mathrm{KG}$ body weight) whereas the dosage used in this study was only $2 \mathrm{mg} / \mathrm{kg}$. It is essential to point out that the $\mathrm{LD}_{50}$ dose for PZ reported is $65 \mathrm{mg} / \mathrm{Kg}$ by Premakumari et al., (1977) 
and $16 \mathrm{mg} / \mathrm{kg}$ b. w. $\left(\mathrm{LD}_{50}\right)$ was reported by SivaKumar et al., (2005). However, the dosage used by them are far high from LD 50 dose in the literature, as well as from the dose used in the present study and hence the results.

\section{CONCLUSION}

Administration of the ethanoloic extract of the $P$. zeylanica (and naphthoquinone) altered the neurotransmitter profile in few specific regions of Wistar rat brain and striatum was found to be affected more.

\section{REFERENCES}

Abdul KM, Ramchender RP. Modulatory effect of plumbagin (5-hydroxy-2-methyl-1,4-naphthoquinone) on macrophage functions in $\mathrm{BALB} / \mathrm{c}$ mice. I. Potentiation of macrophage bactericidal activity. Immunopharmacology. 1995; 30:231-236.

Bopaiah CP, Pradhan N. Central nervous system stimulatory action from the root extract of Plumbago zeylanica in rats. Phytother Res. 2001;15:153-156.

De Paiva SR, Figueiredo MR, Aragao TV, Kaplan MA. Antimicrobial activity in vitro of plumbagin isolated from Plumbago species. MemInst Oswaldo Cruz. 2003;98:959-61.

Edenharder R, Tang X Inhibition of the mutagenicity of 2nitrofluorene, 3-nitrofluoranthene and 1-nitropyrene by flavonoids, coumarins, quinones and other phenolic compounds. Food ChemToxicol. 1997;35:357-372.

Ganong WF. 2001. Synaptic and junctional transmission. Review of medical physiology, $20^{\text {th }}$ edition. The McGraw-Hill companies, Inc. pp110.

Kim C, Campanelli C, Khanna JM. Determination of picogram levels of brain catecholamines and indoles by a simplified liquid chromatographic electrochemical detection method. J Chromatogr. 1983;282:151-159.
Krishnaswamy M, Purushothaman KK. Plumbagin: A study of its anticancer, antibacterial and antifungal properties. Ind J Exp Biol. 1980;18:876-877.

Kumar NB, Hopkins K, Allen K, Riccardi D, Besterman-Dahan $\mathrm{K}$, Moyers S. Use of complementary/integrative nutritional therapies during cancer treatment: implications in clinical practice. Cancer Control. 2002;9:236-243.

Manu Pant, AnkitaLal, Swati Rana, and Anju Rani. Plumbago zeylanica L. : A Mini Review. International Journal of Pharmaceutical Applications. 2012;3(3): 399-405.

Premakumari P, Rathinam K, Santhakumari G. Antifertility activity of plumbagin. Ind J Med Res. 1977;65:829-838.

Santhakumari G and Rathinam. K Anticoagulant activity of Plumbagin. Indian J Exp Biol. 1978;16:485-487.

Sembulingam K, Sembulingam P, Namsivayam A. Effect of Ocimum sanctum Linn on the changes in central cholinergic system induced by acute noise stress. J Ethnopharmacol. 2005;96:477-482.

Siva Kumar V, Prakash R, Murali MR. Invivo micronucleus assay and GST activity in assessing genotoxicity of puumbagin in Swiss albino mice. Drug ChemToxicol. 2005;28:499-507.

Srinivas P, Gopinath G, Banerji A, Dinakar A, Srinivas G. Plumbagin induces reactive oxygen species, which mediate apoptosis in human cervical cancer cells. MolCarcinog. 2004; 40:201-211.

Wagner J, Vitali P, Palfreyman MG, Zariaka M, Zariaka M, Huot S. Simultaneous determination of 3,4-dihydroxy Phenyl alanine, 5hydroxyl tryphtophan, dopamine, 4-hydroxy 3-methoxy phenyl alanine, norepinephrine, 3. 4 dihydroxyphenylacetic acid, homovanillic acid, serotonin and 5 hydroxyindole acetic acid in rat cerebrospinal fluid and brain by High performance Liquid Chromatography with electrochemical detection. J Neurochem. 1982;38:1241-1254.

\section{How to cite this article:}

Baskaran M., Shanmugam L., Raman V. Effect of Plumbago zeylanica administration on brain neurotransmitter level in Wistar albino rats. J App Pharm Sci, 2015; 5 (04): 053-057. 